\title{
THE LOW-DIMENSIONAL METRIC FOLIATIONS OF EUCLIDEAN SPHERES
}

\author{
DETLEF GROMOLL \& KARSTEN GROVE
}

A metric foliation of a Riemannian manifold is a smooth partition into lower dimensional submanifolds which are locally everywhere equidistant, like for example, orbits of an isometric group action, or "parallel" hypersurfaces. In a general setting one has to allow singular focal leaves, but we will only consider the nonsingular case here. The leaves of a metric foliation are locally given as fibers of a Riemannian submersion which projects the induced metric of the normal bundle along the leaves isometrically to a metric on the quotient manifold. Although we are basically just dealing with Riemannian foliations for which the fixed metric is bundlelike [15], understanding the structure of metric foliations is primarily a problem in differential geometry that has a significant local component. Traditionally, in foliation theory only a transversal Riemannian structure is considered to be given on the ambient manifold.

Riemannian submersions satisfy highly overdetermined equations, and they do not exist for generic metrics, except with fibers of codimension 1 . On the other hand, many important metrics, including in particular homogeneous metrics, admit an abundance of (usually nonhomogeneous) local Riemannian submersions with certain fiber dimensions, and sometimes, as in the case of constant curvature, with arbitrary fiber dimension. This makes the study of metric foliations in space forms especially attractive. Surprisingly enough, very little was known until recently.

In [6], we began a systematic investigation of $k$-dimensional metric foliations $\mathscr{F}^{k}$ in a constant curvature space $Q_{c}^{n+k}$, and we gave a complete analysis in the case $k=1$. Here we continue this program and consider higher dimensional foliations. The general situation is quite complex, in particular locally. The global behavior of $\mathscr{F}$ depends critically on the sign of the curvature $c$. Our main result (Theorem 5.3) is concerned with spherical space forms. We classify the metric foliations $\mathscr{F}^{k}$ of the Euclidean sphere $S^{n+k}$ for $k \leq 3$. They are all homogeneous, and moreover orbit foliations arising from a locally free

Received March 6, 1987. This work was partially supported by National Science Foundation grants DMS-8405956 and DMS-8406471. 
orthogonal action of a $k$-dimensional simply connected Lie group. The latter cannot occur whenever $k>3$. The 1-dimensional metric foliations correspond to nonvanishing Killing fields on odd dimensional spheres, 2-dimensional metric foliations do not exist, and there are finitely many 3-dimensional metric foliations on the spheres $S^{4 l+3}$. As a consequence we obtain that the Hopf fibrations are metrically rigid, i.e., they are the only Riemannian submersions of a Euclidean sphere, except possibly for $S^{15} \rightarrow M^{8}$. This has an important application in the context of [7] and [3]. When all fibers are assumed to be totally geodesic, the last result was proved in [4] (cf. also [14] and [18]). Such foliations are in fact already locally rigid, which can be seen most easily by observing that reflections in leaves induce local isometric reflections in points on the base.

We should mention that complete metric foliations tend to have strong rigidity properties if the ambient space has nonnegative curvature, but this is not so in case of negative curvature (cf. [6]). We finally point out another intriguing aspect of this work which emerged already in [6]. We had called a metric foliation in a space of constant curvature isoparametric if the mean curvature form of the leaves is basic, or equivalently, if all principal curvatures with respect to basic normal fields are locally constant along leaves. This notion is considerably more general than that used in the beautiful work on Terng, Hsiang and Palais [17], [9] which in our terminology deals with complete flat metric foliations in Euclidean spaces. Such foliations are essentially always singular. All complete metric foliations in space forms of positive or zero curvature turn out to be isoparametric. Further discussions including local considerations will be contained in [8].

\section{The basic geometry of $\mathscr{F}$}

For sume facts about Riemannian submersions which will be used freely throughout this paper we refer to [13] (cf. also [6]). The discussion in this section is not restricted to constant curvature spaces $Q$ unless otherwise stated. The foliation $\mathscr{F}$ defines an orthogonal splitting $T Q=\Delta^{v} \oplus \Delta^{h}$ of the tangent bundle of $Q$, where $\Delta^{v}$ is the "vertical" tangent bundle along the leaves. We have a corresponding decomposition

$$
\mathfrak{X}_{U} \simeq \mathfrak{X}^{v} \oplus \mathfrak{X}^{h}
$$

of all vector fields on an open subset $U$ of $Q$. Whenever the restriction of $\mathscr{F}$ to $U$ is given by a Riemannian submersion $\pi: U \rightarrow M$, we consider the subspace

$$
\mathfrak{B} \subset \mathfrak{X}^{h}
$$


of basic fields, i.e., horizontal $\pi$-related lifts of fields on $M$, so $\mathfrak{B}$ is canonically isomorphic to $\mathfrak{X}_{M}$. We always use $T$ to denote vertical fields, and $X, Y, Z$ to denote basic fields on $U$ related to $\bar{X}, \bar{Y}, \bar{Z}$ on $M$. Observe that in general

$$
\left[\mathfrak{B}, \mathfrak{X}^{v}\right] \subset \mathfrak{X}^{v}
$$

since $[X, T]^{h}=0$, or equivalently $L_{X} T=[X, T] \in \mathfrak{X}^{v}$. Of course, $\mathfrak{X}^{v}$ is a Lie subalgebra of $\mathfrak{X}_{U}$, whereas $\mathfrak{X}^{h}$ or $\mathfrak{B}$ are typically not subalgebras.

The integrability tensor $A$ of the horizontal bundle $\Delta^{h}$ is the skew 2-form on $\Delta^{h}$ with values in $\Delta^{v}$, given by

$$
A_{X} Y=\frac{1}{2}[X, Y]^{v}=\stackrel{v}{\nabla}_{X} Y
$$

where $\nabla$ is the standard Riemannian connection of $Q$. The foliation is flat if $A \equiv 0$, or equivalently if $\mathfrak{B}, \mathfrak{X}^{h}$ are subalgebras, i.e., $\Delta^{h}$ is integrable. Consider the subspace of integrability fields

$$
\mathscr{A} \subset \mathfrak{X}^{v}
$$

spanned by all fields $A_{X} Y$ on $U$, and the subalgebra $[\mathscr{A}]$ of $\mathfrak{X}^{v}$ generated by $\mathscr{A}$. Note that for any leaf $W$ in $U$, the restriction $\mathscr{A}_{W}$ of $\mathscr{A}$ to $W$ is finite dimensional, in fact

$$
0 \leq \operatorname{dim} \mathscr{A}_{W} \leq\left(\begin{array}{l}
n \\
2
\end{array}\right) \text {. }
$$

The restriction $[\mathscr{A}]_{W}=\left[\mathscr{A}_{W}\right]$ is usually infinite dimensional; we refer to it as the holonomy algebra of $W$.

It follows from (1.1) that

$$
\stackrel{h}{\nabla}_{T} X=\stackrel{h}{\nabla}_{X} T=-A_{X}^{*} T
$$

where $A_{X}^{*}$ is the (pointwise) adjoint of the transformation $A_{X}$. The curvature tensors of $M$ and $U$ always satisfy the relation

$$
\left(R_{M}-R^{h}\right)(X, Y, Z)=A_{X}^{*} A_{Y} Z-A_{Y}^{*} A_{X} Z-2 A_{Z}^{*} A_{X} Y .
$$

In the case of constant curvature, $R(X, Y) Z=c(\langle Y, Z\rangle X-\langle X, Z\rangle Y)$ is basic, and so is therefore the right-hand side of (1.4). In particular, $A_{X}^{*} A_{X} Y$ is basic, hence $\left\langle A_{X} Y, A_{X} Z\right\rangle$ is constant along leaves,

$$
T\left\langle A_{X} Y, A_{X} Z\right\rangle=0 .
$$

The second fundamental form $S$ of $\mathscr{F}$ is the horizontal 1-form on $\Delta^{h}$ with values in selfadjoint transformations of $\Delta^{v}$,

$$
S_{X} T=-\stackrel{v}{\nabla}_{T} X
$$


The mean curvature form is the horizontal 1-form $\kappa$ on $Q$,

$$
\kappa(U)=\operatorname{tr} S_{U^{h}}
$$

\section{Holonomy algebras of substantial foliations}

In this section we show that the space of integrability fields $\mathscr{A}_{W}$ coincides with the holonomy algebra $\left[\mathscr{A}_{W}\right]$ provided the foliation $\mathscr{F}$ is highly nonflat.

Fix a connected open set $U$ on which $\mathscr{F}$ is given by a submersion, as in $\S 1$. Let us call $T_{1}, T_{2} \in \mathfrak{X}^{v}$ congruent modulo $\mathscr{A}$ if $T_{2}-T_{1} \in \mathscr{A}$. In this case we write $T_{1} \sim T_{2}$.

We begin with a general observation. For any $X, Y, Z \in \mathfrak{B}$,

$$
L_{X} A_{Y} Z=\frac{1}{2}\left[X,[Y, Z]^{v}\right]^{v}=\stackrel{v}{\nabla}_{X} A_{Y} Z+S_{X} A_{Y} Z,
$$

using (1.1), (1.2), and (1.6). Thus by the Jacobi identity,

$$
\complement \stackrel{v}{\nabla}_{X} A_{Y} Z+\complement S_{X} A_{Y} Z=\complement L_{X} A_{Y} Z=-\complement A_{X}[Y, Z]^{h} \sim 0,
$$

where $\complement$ denotes cyclic summation. Equality holds everywhere if $\bar{X}, \bar{Y}, \bar{Z}$ commute mutually on the local quotient. We now restrict attention to the case of constant curvature.

Lemma 2.3. For all fields $X, Y, Z \in \mathfrak{B}$,

$$
\stackrel{v}{\nabla}_{X} A_{Y} Z-\stackrel{v}{\nabla}_{Y} A_{X} Z \sim-2 S_{Z} A_{X} Y .
$$

Proof. From $\stackrel{v}{\nabla}_{X} A_{Y} Z=\stackrel{v}{\nabla} \stackrel{v}{\nabla}_{Y} Z=\stackrel{v}{\nabla}_{X} \nabla_{Y} Z-\stackrel{v}{\nabla}_{X} \stackrel{h}{\nabla}_{Y} Z=\stackrel{v}{\nabla}{ }_{X} \nabla_{Y} Z-$ $A_{X} \stackrel{h}{\nabla}_{Y} Z$ and $R^{v}(X, Y) Z=0$ we obtain

$$
\begin{aligned}
\stackrel{v}{\nabla}_{X} A_{Y} Z-\stackrel{v}{\nabla}_{Y} A_{X} Z & =\stackrel{v}{\nabla}_{[X, Y]} Z-A_{X} \stackrel{h}{\nabla_{Y}} Z+A_{Y} \stackrel{h}{\nabla}_{X} Z \\
& =\stackrel{v}{\nabla}_{[X, Y]^{v}} Z+\stackrel{v}{\nabla}_{[X, Y]^{h}} Z-A_{X} \stackrel{h}{\nabla}_{Y} Z+A_{Y} \stackrel{h}{\nabla}_{X} Z .
\end{aligned}
$$

Hence,

$$
\stackrel{v}{\nabla}_{X} A_{Y} Z-\stackrel{v}{\nabla}_{Y} A_{X} Z=-2 S_{Z} A_{X} Y+A_{[X, Y]^{h}} Z-A_{X} \stackrel{h}{\nabla}_{Y} Z+A_{Y} \stackrel{h}{\nabla}_{X} Z
$$

Lemma 2.5. For all fields $X, Y, Z \in \mathfrak{B}$,

$$
\stackrel{v}{\nabla}_{Z} A_{X} Y \sim S_{Z} A_{X} Y-S_{X} A_{Y} Z-S_{Y} A_{Z} X
$$

In particular,

$$
\stackrel{v}{\nabla}_{X} A_{X} Y \sim 2 S_{X} A_{X} Y
$$


Proof. Applying (2.2) and (2.4) to the identity

$$
\stackrel{v}{\nabla}_{Z} A_{X} Y=\complement \stackrel{v}{\nabla}{ }_{X} A_{Y} Z-\left(\stackrel{v}{\nabla}_{X} A_{Y} Z-\stackrel{v}{\nabla}_{Y} A_{X} Z\right)
$$

yields

$$
\stackrel{v}{\nabla}{ }_{Z} A_{X} Y=S_{Z} A_{X} Y-S_{X} A_{Y} Z-S_{Y} A_{Z} X+A_{X} \stackrel{h}{\nabla}{ }_{Z} Y-A_{Y} \stackrel{h}{\nabla}{ }_{Z} X .
$$

For future reference we note

$$
L_{Z} A_{X} Y \sim 2 S_{Z} A_{X} Y-S_{X} A_{Y} Z-S_{Y} A_{Z} X
$$

as an immediate consequence of (2.1) and (2.5).

If $X$ is the tangent field of a horizontal geodesic $\gamma$, and $Y$ is horizontally parallel along $\gamma$, then we conclude from (2.6),

$$
\stackrel{v}{\nabla}_{X} A_{X} Y=2 S_{X} A_{X} Y \text {. }
$$

In particular, the kernel of $A_{X}$ is horizontally parallel, and $A_{X}$ has constant rank along $\gamma$.

Corollary 2.9. For all $X, Y, Z \in \mathfrak{B},\left\langle S_{X} A_{X} Y, A_{X} Z\right\rangle$ is constant along leaves.

Proof. Using (2.6), we obtain

$$
2\left\langle S_{X} A_{X} Y, A_{X} Y\right\rangle=\frac{1}{2} X\left\|A_{X} Y\right\|^{2}-\left\langle A_{X} \stackrel{h}{\nabla}_{X} Y, A_{X} Y\right\rangle-\left\langle A_{Y} \nabla_{X} X, A_{Y} X\right\rangle,
$$

which is constant along leaves by (1.5). The claim follows by polarization.

We call $\mathscr{F}$ substantial along a leaf $\mathscr{F}_{p}$ if $A_{X}$ maps $\Delta^{h}$ onto $\Delta^{v}$ for some normal vector $X$ (and thus for an open dense subset of normal vectors) at some point $q \in \mathscr{F}_{p}$, or equivalently, if $A_{X}^{*}$ is injective. This condition is independent of $q$, since the dimension of the kernel of $A_{Z}$ is constant for local basic fields $Z$, by (1.5). It follows then from (2.8) (applied to broken horizontal geodesics) that $A$ is substantial along all leaves in an open dense subset, since $Q$ is connected.

Lemma 2.10. If $\mathscr{F}$ is substantial along all leaves in $U$, then

$$
S_{X} A_{X} Y \sim 0 \text { for } X, Y \in \mathfrak{B} \text {. }
$$

Proof. We show that $S_{X} A_{X} Y_{\mid W} \in \mathscr{A}_{W}$ for any leaf $W$ in $U$. It suffices to assume $A_{X}$ is onto along $W$. Then using (1.5) and Corollary 2.9, we find $Y_{1}, \cdots, Y_{k} \in \mathfrak{B}$ such that $A_{X} Y_{1}, \cdots, A_{X} Y_{k}$ are orthonormal fields of eigenvectors for $S_{X}$ with constant eigenvalues $\lambda_{1}, \cdots, \lambda_{k}$ along $W$. Thus $S_{X} A_{X} Y=A_{X} Z$ along $W$ for some $Z \in \mathfrak{B}$.

Remark 2.11. The proof of Lemma 2.10 has another interesting consequence: If $\mathscr{F}$ is substantial along some leaf, then the principal curvatures of $S$ are constant for all (local) basic $X$ along any leaf, i.e., $\mathscr{F}$ is isoparametric. 
Lemma 2.12. Suppose $\mathscr{F}$ is substantial along all leaves in $U$. Then

$$
L_{X} A_{Y} Z \sim 0 \text { for } X, Y, Z \in \mathfrak{B} .
$$

Proof. According to (2.7) and Lemma 2.10 we have $L_{X} A_{X} Y \sim 3 S_{X} A_{X} Y$ $\sim 0$, and $L_{X} A_{Y} Z$ is skew symmetric in all three arguments, up to congruence. Therefore, $L_{X} A_{Y} Z \sim L_{Y} A_{Z} X \sim L_{Z} A_{X} Y$, and hence $0 \sim \complement L_{X} A_{Y} Z \sim$ $3 L_{X} A_{Y} Z$ by (2.2). This completes the proof.

Proposition 2.13. Suppose $\mathscr{F}$ is substantial along all leaves $W$ in $U$. Then $\mathscr{A}=[\mathscr{A}]$ and thus $\mathscr{A}_{W}=\left[\mathscr{A}_{W}\right]$ are Lie algebras, $k \leq \operatorname{dim} \mathscr{A}_{W} \leq\left(\begin{array}{c}n \\ 2\end{array}\right)$. Furthermore, $\mathscr{A} \oplus \mathfrak{B}$ is a Lie algebra containing $\mathscr{A}$ as an ideal.

Proof. Let $T \in \mathscr{A}$ and $X, Y \in \mathfrak{B}$. Then

$$
2\left[A_{X} Y, T\right]=L_{[X, Y]^{v}} T=L_{[X, Y]} T-L_{[X, Y]^{h}} T=\left[L_{X}, L_{Y}\right] T-L_{[X, Y]^{h}} T,
$$

and all assertions follow immediately from Lemma 2.12.

\section{Local homogeneity aspects}

The foliation $\mathscr{F}$ is called homogeneous if there is an isometric group action on $Q$ preserving $\mathscr{F}$ such that the isotropy group of each leaf $\mathscr{F}_{p}$ is transitive on $\mathscr{F}_{p}$ (cf. also [12] in this context). If near any point we only have such actions by local groups of isometries, we say $\mathscr{F}$ is locally homogeneous.

In this section, we will show that low dimensional substantial foliations in space forms are locally homogeneous in a strong sense. Let us restrict $\mathscr{F}$ again to a suitable open set $U$ as before. In the next three lemmas we assume that $\mathscr{F}$ is substantial along a leaf $W$ in $U$ and $\operatorname{dim} W=k \leq 3$.

Lemma 3.1. Let $T_{1}, T_{2} \in \mathscr{A}_{W}$. Then $\left\langle T_{1}, T_{2}\right\rangle$ is constant on $W$. In particular, $\operatorname{dim} \mathscr{A}_{W}=k$.

Proof. It suffices to prove

$$
\left\langle A_{X} Y, A_{Z} V\right\rangle \text { is constant on } W,
$$

for arbitrary $X, Y, Z, V \in \mathfrak{B}$.

We only have to consider the cases $k=2$ and $k=3$, which we treat simultaneously. First suppose, $H$ is a subspace of basic fields along $W, 3 \leq$ $\operatorname{dim} H=m+1 \leq 4, m=2$ only when $k=2$ and $n=3$, such that for some $X_{0} \in H$, the vectors $A_{X_{0}} Y, Y \in H$, span $\Delta^{v}$ at some point, and thus along $W$ by (1.5). Then (3.2) holds for all $X, Y, Z, V \in H$. To see this, choose independent $X_{l} \in H, 0 \leq l \leq m$, with $A_{X_{0}} X_{l}$ orthonormal for $1 \leq l \leq k$. We are done if we show $\left\langle A_{X_{i}} X_{j}, A_{X_{0}} X_{l}\right\rangle$ is constant for $0 \leq i<j \leq m, 1 \leq l \leq k$, observing the skew symmetry of $A$. But by (1.5), the last claim follows for $i=0$, or $i=l$, or $j=l$. The remaining cases are then obtained using that 
$A_{X_{i}} X_{j}$ has constant length. Since $A$ is substantial along $W$, a straightforward limiting argument now yields (3.2) for arbitrary $X, Y, Z, V \in \mathfrak{B}$.

According to Proposition 2.13 and Lemma $3.1, \mathscr{A}_{W}$ is a $k$-dimensional Lie algebra of vector fields, usually not Killing. However, we can construct local isometries of $W$ by changing the local action of the corresponding Lie group from "left" to "right".

Lemma 3.3. For each $p \in W$ there is an open neighborhood $\Omega$ in $W$ and a unique $k$-dimensional Lie algebra $\mathscr{K}_{\Omega}$ of Killing fields on $\Omega$ such that $\left[\mathscr{K}_{\Omega}, \mathscr{A}_{\Omega}\right]=0$. The associated local isometric Lie group action is free.

Proof. Consider the local Lie group $G$ of local diffeomorphisms of $W$ near $p$, generated by $\mathscr{A}_{W}$. By Lemma 3.1 , we can choose an open neighborhood $\mathscr{O}$ of the identity in $G$ such that the left action $P: \mathscr{O} \rightarrow W, P(g)=g(p)$, maps $\mathscr{O}$ diffeomorphically onto the orbit $P(\mathscr{O})=\Omega$. The induced Lie algebra isomorphism $P_{*}: \mathfrak{X}_{\mathscr{Q}} \rightarrow \mathfrak{X}_{\Omega}$ carries the subalgebra of right invariant fields $\mathscr{R}_{\mathscr{O}}$ onto $\mathscr{A}_{\Omega}$. Let $\mathscr{L}_{\mathscr{O}}$ denote the left invariant fields, and set $\mathscr{K}_{\Omega}=P_{*} \mathscr{L}_{\mathscr{O}}$. Clearly, the algebras $\mathscr{K}_{\Omega}$ and $\mathscr{A}_{\Omega}$ commute. Since $\mathscr{A}_{\Omega}$ contains a basis of pointwise orthonormal fields according to (1.5), it follows that each $T \in \mathscr{K}_{\Omega}$ is a Killing field. The remaining assertions are straightforward.

Our next step is to extend the fields $\mathscr{K}_{\Omega}$ of Lemma 3.3 to Killing fields near $\Omega$ in $U$.

Lemma 3.4. Let $\Omega$ be as in Lemma 3.3 and $U$ a tubular neighborhood of $\Omega$ in $Q$. Then $\mathscr{K}_{\Omega}$ extends to a unique algebra $\mathscr{K}$ of (vertical) Killing fields on $U$ such that $\mathscr{K}_{\Omega^{\prime}}=\mathscr{K}_{\Omega^{\prime}}$ for each substantial leaf $\Omega^{\prime}$ in $U$. In particular, $\mathscr{F}$ restricted to $U$ is locally homogeneous.

Proof. Let $h$ be a local isometry in $\Omega$ generated by some field in $\mathscr{K}_{\Omega}$. Extend $h$ to an orthogonal isomorphism $H$ of normal bundles along $\Omega$ in $Q$, Bott parallel, i.e. $H X=X \circ h$ for $X \in \mathfrak{B}$.

We claim that $H$ preserves the normal connection and the second fundamental form. Let $T \in \mathscr{A}, X \in \mathfrak{B}$. Then $\stackrel{h}{\nabla}_{T} X=-A_{X}^{*} T \in \mathfrak{B}$ by (1.3) and Lemma 3.1, and $h_{*} T=T \circ h$ by Lemma 3.3. Now the first assertion is obtained from the following calculation along $h$ :

$$
\begin{aligned}
\stackrel{h}{\nabla}_{T} H X & =\stackrel{h}{\nabla}_{T} X \circ h=\stackrel{h}{\nabla}_{h_{*} T} X=\stackrel{h}{\nabla}_{T \circ h} X \\
& =-\left(A_{X}^{*} T\right) \circ h=-H A_{X}^{*} T=H \stackrel{h}{\nabla}_{T} X .
\end{aligned}
$$

To prove the second assertion, observe in addition $S_{X} \mathscr{A} \subset \mathscr{A}$, in view of Lemmas 2.10 and 3.1. Thus

$$
h_{*} S_{X} T=\left(S_{X} T\right) \circ h=S_{X \circ h} T \circ h=S_{H X} h_{*} T .
$$


Now we can apply the Fundamental Theorem for submanifolds (cf. [16] for example). It follows that $h$ extends to a local isometry in $U$ given by $\exp _{\Omega} \circ H \circ \exp _{\Omega}^{-1}$, where $\exp _{\Omega}$ is the normal exponential map of $\Omega$ in $U$. By construction, these extensions preserve the leaves in $U$ and define the algebra $\mathscr{K}$ uniquely.

Since the substantial leaves form an open and dense subset of $Q$, the following conclusion is an immediate consequence of the results in this section.

Proposition 3.5. Suppose $\mathscr{F}$ is substantial along some leaf, $\operatorname{dim} \mathscr{F} \leq 3$. Then $\mathscr{F}$ is locally homogeneous and in fact everywhere given by (local) free isometric group actions.

\section{Restrictions in the complete case}

The main purpose of this section is to show that in a complete space $Q_{c}$ with $c>0$ a (nonsingular) metric foliation $\mathscr{F}^{k}, k \leq 3$, is substantial. In general, there is no such restriction for $c \leq 0$.

We begin with some simple remarks concerning holonomy. Recall that since $\mathscr{F}$ is locally described in terms of Riemannian submersions, any piecewise differentiable horizontal curve $\gamma:[a, b] \rightarrow Q$ extends to a unique family of nearby horizontal curves with same projection in local quotients. This way, $\gamma$ gives rise to a diffeomorphism $h^{\gamma}$ of a neighborhood of $\gamma(a)$ in the leaf $\mathscr{F}_{\gamma(a)}$ onto a neighborhood of $\gamma(b)$ in $\mathscr{F}_{\gamma(b)}$, the "holonomy displacement" along $\gamma$. In particular, let $\gamma$ be the restriction of a unit speed geodesic $\gamma_{X}$ to $[0, t]$, $\dot{\gamma}_{X}=X$, and $h^{t}=h^{\gamma}$. Then the differential of $h^{t}$ is given by the Jacobi fields $T$ along $\gamma$ in $Q$,

$$
h_{*}^{t} T_{0}=T(t) \quad \text { with } T(0)=T_{0}, T^{\prime}(0)=-S_{X} T_{0}-A_{X}^{*} T_{0} .
$$

For the last equality we have used (1.3) and (1.6).

Being a local diffeomorphism, the differential $h_{*}^{\gamma}$ of any holonomy displacement $h^{\gamma}$ is always nonsingular. Therefore, the Jacobi fields in (4.1) have no zeros unless they vanish identically. In the remaining part of this section, $\mathscr{F}$ will always be a (nonsingular) metric foliation of a complete $Q_{c}$. In this case we can immediately draw the following conclusion from the above.

Lemma 4.2. If $S_{X} T_{0}=\lambda T_{0}, T_{0} \neq 0$, and $\lambda^{2}+c>0$, then $A_{X}^{*} T_{0} \neq 0$.

This lemma shows that the integrability tensor $A$ is nontrivial everywhere for $c>0$, and also for $c=0$ unless $\mathscr{F}$ is locally congruent to the product foliation $\mathbf{R}^{k} \times \mathbf{R}^{n}$ (cf. also [6], [8]). Moreover Lemma 4.2 is also the key to our crucial

Proposition 4.3. Let $\mathscr{F}$ be a $k$-dimensional metric foliation of a complete $Q_{c}^{k+n}$. If $c>0$ and $k \leq 3$, then $A$ is substantial along all leaves. 
Proof. By passing to the universal cover we can assume, after rescaling the metric, that $Q=S^{k+n}$. First observe that $k+n$ must be odd, since the tangent bundle of an even-dimensional sphere cannot split. Now fix a point $p \in S^{k+n}$.

If the linear map $X \rightarrow S_{X}$ has a nontrivial kernel, the claim follows from Lemma 4.2. Therefore, we assume that $S$ is injective, so in particular $n \leq$ $k(k+1) / 2$, and it remains to discuss the cases when $(k, n)$ is one of the pairs $(2,3),(3,6),(3,4),(2,1),(3,2)$. Although we have to deal with each situation individually, the arguments will always use Lemma 4.2 in an essential way.

In the first three cases we will make use of the following general facts. Consider for $T \in \Delta_{p}^{v}=\mathbf{R}^{k}$ the skew symmetric transformation $A_{T}$ of $\Delta_{p}^{h}=$ $\mathbf{R}^{n}$ defined by

$$
A_{T} X=A_{X}^{*} T
$$

Then we have for $T \neq 0$,

$$
\text { rk } A_{T}>n-k \text {. }
$$

In particular, whenever $n \geq k$,

$$
\operatorname{dim} E=k, \quad E=\operatorname{im}\left(T \rightarrow A_{T}\right) .
$$

We note that (4.5) is equivalent to saying that $\Delta_{p}^{v}$ is spanned by all vectors $A_{X} Y$. To prove (4.4), suppose $\mathrm{rk} A_{T} \leq n-k$, or equivalently, $\operatorname{dim} \operatorname{ker} A_{T_{0}} \geq k$ for some $T_{0} \neq 0$. Since the space of selfadjoint transformations of $\Delta_{p}^{v}$ with eigenvector $T_{0}$ has codimension $k-1$, we find $0 \neq X_{0} \in \operatorname{ker} A_{T_{0}}$ such that $T_{0}$ is an eigenvector of $S_{X_{0}}$, contradicting Lemma 4.2 .

Assume now that $A$ is not substantial along $\mathscr{F}_{p}$. This means $E$ in (4.5) is an annihilator space of $\Delta_{p}^{h}=\mathbf{R}^{n}$, i.e. for any $x \in \mathbf{R}^{n}$ there exists $0 \neq a \in E$ with $a x=0$. However, by Lemma 4.2 , no $x \neq 0$ is annihilated by all of $E$. Therefore,

$$
1 \leq \operatorname{dim}\{a \in E \mid a x=0\}<k
$$

for all $x \neq 0$.

Let $k=2$ and $n=3$. The claim follows from (4.5), since clearly no proper subspace of the skew symmetric transformations of $\mathbf{R}^{3}$ annihilates $\mathbf{R}^{3}$.

Now let $k=3$ and $n=6$. By (4.4), $\operatorname{dim}$ ker $a \leq 2$ for all $0 \neq a \in E$. Therefore $E$ cannot annihilate $\mathbf{R}^{6}$ for dimension reasons, since $\operatorname{dim} E=3$.

In the tightest case $k=3$ and $n=4$, we proceed as follows. Again by (4.4), $\operatorname{rk} a \geq 2$ for all $0 \neq a \in E$. Let $\bar{a}$ denote the 2 -form on $\mathbf{R}^{4}$ associated to $a$. Note that $\mathrm{rk} a=2$ iff $\bar{a} \wedge \bar{a}=0$. Suppose first that there exists $a_{0} \in E$ which is invertible, i.e., the quadratic form $f$ on $E, f(a)=\bar{a} \wedge \bar{a}$, is nontrivial. Therefore, $f^{-1}(0)$ is a cone in $E$ over a manifold of dimension $\leq 1$. We 
conclude that $f^{-1}(0)$ and thus $E$ can only annihilate a set of dimension $\leq 3$ in $\mathbf{R}^{4}$. It remains to show $f \equiv 0$ is impossible under our assumptions. In that situation, all $\bar{a}$ are decomposable and $\bar{a} \wedge \bar{b}=0$ for $a, b \in E$. Hence $\bar{a}, \bar{b}$ have a common factor, i.e., $\bar{a}=\rho \wedge \alpha, \bar{b}=\rho \wedge \beta$. Now choose a basis $a_{1}, a_{2}, a_{3}$ of $E$ and write

$$
\bar{a}_{1}=\varepsilon_{0} \wedge \varepsilon_{1}, \quad \bar{a}_{2}=\varepsilon_{0} \wedge \varepsilon_{2},
$$

with independent 1 -forms $\varepsilon_{0}, \varepsilon_{1}, \varepsilon_{2}$ on $\mathbf{R}^{4}$. Suppose, $\bar{a}_{3}$ is not contained in the span of $\varepsilon_{i} \wedge \varepsilon_{j}, 0 \leq i<j \leq 2$. Then we find $\varepsilon_{3}$ such that $\bar{a}_{3}=\left(s_{1} \varepsilon_{0}+t_{1} \varepsilon_{1}\right) \wedge$ $\varepsilon_{3}=\left(s_{2} \varepsilon_{0}+t_{2} \varepsilon_{2}\right) \wedge \varepsilon_{3}$. We conclude $s_{1}=s_{2}=s, t_{1}=t_{2}=0$. But then $\bar{a}_{3}=s \varepsilon_{0} \wedge \varepsilon_{3}$, and no $0 \neq a \in E$ annihilates the vector $e_{0}$ of the dual basis $e_{0}, e_{1}, e_{2}, e_{3}$ of $\mathbf{R}^{4}$. Otherwise, choose any $\varepsilon_{3}$ independent of $\varepsilon_{0}, \varepsilon_{1}, \varepsilon_{2}$. Now $E$ annihilates $e_{3}$, contradicting (4.6). In the latter situation, $E$ is essentially the space of all skew symmetric transformations of a 3-dimensional subspace of $\mathbf{R}^{4}$.

The last two cases $(2,1),(3,2)$ actually do not occur, as a consequence of the next general result.

Theorem 4.7. There are no metric foliations of $Q_{c}^{k+n}$ for $c>0$ with codimension $n \leq 2$, except when $k=1$ and $n=2$.

Proof. By Lemma 4.2, this is immediately clear in the flat case, i.e. $A \equiv 0$, so in particular for $n=1$. Actually, all of Theorem 4.7 is a consequence of a general result on Riemannian foliations (cf. [11]). In the remaining case of interest to us here, $k=3$ and $n=2$, we give a simple direct proof. Fix a point $p$. All vectors $A_{X} Y$ form a 1-dimensional subspace of $\Delta_{p}^{v}$. By Lemma 4.2 , it suffices to find $X_{0} \neq 0$ so that $S_{X_{0}}$ has an eigenvector perpendicular to the image of $A$. But this follows from the claim that given any 2-dimensional subspace $E$ of all symmetric $3 \times 3$ matrices and any plane $H$ through 0 in $\mathbf{R}^{3}$, there exists $S_{0} \in E^{*}=E \backslash 0$ having an eigenvector in $H$. If not, all $S$ in $E^{*}$ must have distinct eigenvalues $\lambda_{1}<\lambda_{2}<\lambda_{3}$, which are continuous functions and define a continuous frame field of corresponding eigenvectors $e_{1}, e_{2}, e_{3}$ on $E^{*}$ in a fixed open half-space with boundary $H$. Now $e_{1}(-S)=e_{3}(S)$, $e_{2}(-S)=e_{2}(S), e_{3}(-S)=e_{1}(S)$. This is impossible since $e_{3}, e_{2}, e_{1}$ and $e_{1}, e_{2}, e_{3}$ represent opposite orientations, and $E^{*}$ is connected.

\section{Global conclusions}

Recall that a simply connected space $Q_{c}^{k+n}$ admits an isometric immersion into $M_{c}$, the complete simply connected $(k+n)$-dimensional space with constant curvature $c$. This "developing map" is unique up to congruence. Any connected Lie subgroup $G$ of the isometry group $I_{c}$ of $M_{c}$ gives rise to the 
metric orbit foliation $\mathscr{F}_{G}$ of the open dense subset of points whose isotropy groups have minimal dimension. We can now globalize Proposition 3.5.

Theorem 5.1. Let $\mathscr{F}$ be a $k$-dimensional metric foliation of a simply connected $Q_{c}, k \leq 3$. Suppose $\mathscr{F}$ is substantial along some leaf. Then there exists a connected $k$-dimensional Lie subgroup $G$ of $I_{c}$, acting locally freely on $\mathscr{F}_{G}$, such that $\mathscr{F}$ is the pullback of $\mathscr{F}_{G}$ under the developing map. Thus if $Q$ is complete, i.e. $Q_{c} \simeq M_{c}$, then $G$ acts locally freely everywhere.

Proof. This is again a straightforward application of our discussion in $\S 3$. In particular, we can make use of monodromy based on the uniqueness statement in Lemma 3.4. Observe that any local Killing field in $M_{c}$ has a global extension.

As an immediate consequence of Proposition 4.3 and Theorem 5.1 we have

Corollary 5.2. Any metric foliation $\mathscr{F}^{k}$ of the Euclidean sphere $S^{n+k}$ is homogeneous for $k \leq 3$. In fact, $\mathscr{F}$ is the orbit foliation $\mathscr{F}_{G}$ of a connected $k$-dimensional Lie subgroup $G$ of $S O(n+k+1)$.

We now discuss the classification of the foliations $\mathscr{F}^{k}$ in Corollary 5.2. If $k=1$, the universal cover $\tilde{G} \simeq \mathbf{R}$ is the one-parameter subgroup generated by a nowhere vanishing Killing field. This result was already obtained in [6]. Such foliations exist precisely for even $n$. Up to congruence, they are completely described by diagonalizing the action of $\mathbf{R}$ on $\mathbf{R}^{n+2} \simeq \mathbf{C}^{s}$ as the direct sum $e^{2 \pi i \theta_{1} t} \oplus \cdots \oplus e^{2 \pi i \theta_{s} t}$ of irreducible 2-dimensional representations such that $0<\theta_{1} \leq \cdots \leq \theta_{s}=1$. Note that there are always at least $s=n / 2+1$ compact leaves which are great circles. All orbits are compact iff all $\theta$ 's are rational, i.e., $\mathscr{F}$ is given by a circle action. Among these Seifert fibrations only one is an actual fibration. This is the Hopf fibration corresponding to $\theta_{1}=\cdots=\theta_{s}=1$.

For $k>1$ it is known in general that Riemannian foliations $\mathscr{F}^{k}$ of a simply connected rational homology sphere have only compact leaves, and the generic leaf is an odd-dimensional rational homology sphere [5]. In our situation we can give a complete metric classification. First recall that there is no locally free isometric $\mathbf{R}^{2}$-action on a Euclidean sphere. (This follows directly by diagonalization.) Now let $k=2$. Since a 2-dimensional Lie subalgebra of the orthogonal algebra is necessarily abelian, we conclude that 2-dimensional metric foliations of Euclidean spheres do not exist. This is actually true in simply connected rational homology spheres [5].

The last argument also shows that in the case $k=3, G$ must be a compact Lie group of rank 1 . Thus $\tilde{G} \simeq S U(2)$, and as in the one-dimensional situation, our classification is carried out via representation theory. We refer to [1] and [10] for some well-known results and add brief comments on some facts which may not be so explicit in the literature. 
The action of $\tilde{G}$ decomposes as an orthogonal direct sum $\varphi_{1} \oplus \cdots \oplus \varphi_{s}$ of real irreducible representations $\varphi$ of $S U(2)$ on linear subspaces $E$ in $\mathbf{R}^{n+k+1}$. In our situation, each $\varphi$ must induce a locally free action on the unit sphere in $E$. If an irreducible orthogonal representation is not already unitary for some complex structure, then its complexification is an irreducible unitary representation.

The nontrivial irreducible unitary representations of $S U(2)$ are exactly the symmetric powers $\rho_{m}$ of the standard action $\rho$ of $S U(2)$ on $\mathbf{C}^{2}$ for arbitrary $m \geq 1$. Explicitly, let $V_{m}$ denote the space of homogeneous polynomials $p$ of degree $m$ in two complex variables,

$$
p\left(z_{1}, z_{2}\right)=\sum_{r=0}^{m} a_{r} z_{1}^{r} z_{2}^{m-r} .
$$

Then $\rho_{m}$ is the representation on $V_{m}$ induced by the canonical action of $S U(2)$ on the domain $\mathbf{C}^{2} \simeq V_{1}$. If a real structure, i.e., a complex conjugate linear automorphism $\mathscr{J}$ of $V_{m}$ with $\mathscr{J}^{2}=$ id, commutes with the action $\rho_{m}$, it follows easily that

$$
\mathscr{J} p_{r}=(-1)^{m-r} \omega p_{m-r},
$$

on the basis of monomials $p_{r}=z_{1}^{r} z_{2}^{m-r}, 0 \leq r \leq m$, for some fixed unimodular $\omega$. Therefore, $\rho_{m}$ is the complexification of an irreducible orthogonal representation iff $m$ is even. The maximal torus

$$
T=\left(\begin{array}{rc}
e^{i t \theta} & 0 \\
0 & e^{-i t \theta}
\end{array}\right)=S^{1} \subset S U(2),
$$

and then also $S U(2)$, acts locally freely on $V_{m}$ iff $m$ is odd, as can be seen by a direct computation. Moreover, in this case, the principal orbit is $S U(2) \simeq S^{3}$; points in $S U(2) \cdot p_{r}$ have nontrivial isotropy groups, which are cyclic of order $m-2 r, 0 \leq 2 r<m-1$. In fact, all isotropy groups are cyclic. There are no totally geodesic orbits for $m>1$, since $\rho_{m}$ is irreducible.

We conclude from the above that metric foliations $\mathscr{F}^{3}$ of $S^{n+3}$ exist precisely when $n=4 l$. In any given dimension $4 l+3$, these foliations are, up to congruence, in one-to-one correspondence with direct sums $\rho_{m_{1}} \oplus \cdots \oplus \rho_{m_{s}}$ of complex irreducible representations of $S U(2)$ such that $m_{\sigma}$ is odd and $1 \leq m_{1} \leq \cdots \leq m_{s}, m_{1}+\cdots+m_{s}=2(l+1)-s$. All $\mathscr{F}^{3}$ are generalized Seifert fibrations without totally geodesic leaves iff all $m_{\sigma}>1$. Only one is an actual fibration. This is the Hopf fibration corresponding to $m_{1}=\cdots=m_{l+1}=1$.

We summarize our last results:

Theorem 5.3. Let $\mathscr{F}^{k}$ be a metric foliation of the Euclidean sphere $S^{n+k}$. Then, up to congruence: 
(i) Any $\mathscr{F}^{1}$ is given uniquely by a direct sum of rotations

$$
e^{2 \pi i \theta_{1} t} \oplus \cdots \oplus e^{2 \pi i \theta_{s} t}
$$

where $n=2 s-1$ and $0 \leq \theta_{1} \leq \cdots \leq \theta_{s}=1$.

(ii) $\mathrm{No}_{\mathrm{F}} \mathscr{F}^{2}$ exists.

(iii) Any $\mathscr{F}^{3}$ is given uniquely by a direct sum of irreducible unitary representations $\rho_{m_{1}} \oplus \cdots \oplus \rho_{m_{s}}$ of $S U(2)$, where all $m_{\sigma}$ are odd, $n=4 l$ and $1 \leq m_{1} \leq \cdots \leq m_{s}, m_{1}+\cdots+m_{s}=2(l+1)-s$.

Topologically any fibration of a homotopy sphere $S^{m}$ must have fibers whose homotopy type is $S^{1}, S^{3}$, or $S^{7}$ [2]. The last case is only possible for $m=15$.

Corollary 5.4. Any Riemannian fibration $S^{n+k} \rightarrow M^{n}$ of a Euclidean sphere is congruent to a Hopf fibration, except possibly $S^{15} \rightarrow M^{8}$.

\section{References}

[1] T. Bröcker \& T. t. Dieck, Representations of compact Lie groups, Graduate Texts in Math., Vol. 98, Springer, Berlin, 1985.

[2] W. Browder, Higher torsion in $H$-spaces, Trans. Amer. Math. Soc. 108 (1963) 353-375.

[3] O. Durumeric, A generalization of Berger's theorem on almost 1/4-pinched manifolds. II, J. Differential Geometry 26 (1987) 101-139.

[4] R. H. Escobales, Jr., Riemannian submersions with totally geodesic fibers, J. Differential Geometry 10 (1975) 253-276.

[5] E. Ghys, Feuilletages riemanniens sur les variétés simplement connexes, Ann. Inst. Fourier (Grenoble) 34 (1984) 203-223.

[6] D. Gromoll \& K. Grove, One-dimensional metric foliations in constant curvature spaces, Differential Geometry and Complex Analysis, H. E. Rauch memorial volume, Springer, Berlin, 1985, 165-167.

[7] __ A generalization of Berger's rigidity theorem for positively curved manifolds, Ann. Sci. École Norm. Sup. 20 (1987) 227-239.

[8] _ Metric and isoparametric foliations in space forms, in preparation.

[9] W.-Y. Hsiang, R. S. Palais \& C.-L. Terng, The topology of isoparametric submanifolds, J. Differential Geometry 27 (1987) 423-460.

[10] A. W. Knapp, Representation theory of semi-simple groups, Princeton University Press, Princeton, NJ, 1986.

[11] P. Molino, Geometrie globale des feuilletages riemanniens, Nederl. Akad. Wetensch., A85(1) et Indag. Math. 44 (1) (1982) 45-76.

[12] T. Nagano, Homogeneous sphere bundles and the isotropic riemannian manifolds, Nagoya Math. J. 15 (1959) 29-55.

[13] B. O'Neill, The fundamental equation of a submersion, Michigan Math. J. 13 (1966) 459-469.

[14] A. Ranjan, Riemannian submersions of spheres with totally geodesic fibers, Osaka J. Math. 22 (1985) 243-260.

[15] B. L. Reinhart, Differential geometry of foliations: The fundamental integrability problem, Ergebnisse Math. u. i. Grenzgeb., Vol. 99, Springer, Berlin, 1983. 
[16] M. Spivak, A comprehensive introduction to differential geometry, Vol. 4, Publish or Perish, Boston, 1975.

[17] C.-L. Terng, Isoparametric submanifolds and their Coxeter groups, J. Differential Geometry 21 (1985) 79-107.

[18] J. A. Wolf, Elliptic spaces in Grassmann manifolds, Illinois J. Math. 7 (1963) 425-462.

STATE UNIVERSITY OF NEW YORK, STONY BROOK UNIVERSITY OF MARYLAND 